\title{
Can Bacteria Weather Ammunition Lead Pellets?
}

\author{
A. POTYSZ, Ł. BINKOWSKI ${ }^{2}$, J. KIERCZAK'
}

University of Wroclaw, anna.potysz@uwr.edu.pl

Pedagogical University of Krakow, ljbinkowski@gmail.com

3University of Wroclaw, jakub.kierczak@uwr.edu.pl

Waterfowl hunting pose a risk to the environment, because after the shot, only a few pellets from ammunition cartdridges reach the target bird, while the rest fall on the ground or into the water. Then, spent pellets probably undergo (bio)weathering processes potentially leading to a release of toxic elements including $\mathrm{Pb}, \mathrm{As}$ and $\mathrm{Sb}$. The aim of this study was to evaluate the rate and intensity of pellets (bio)weathering. Experimental dissolution under biotic and abiotic conditions during 60 days was investigated using: i) sediment extract favoring microbial growth (biostimulation), ii) sterile sediments extracts, iii) bacteria Acidithiobacillus thiooxidans that mimicked the extremely acidic conditions that may develop during intense microbial activity, iv) sterile acidic medium and v) root exudates to represent rhizosphere mediated weathering. The risk of metal mobilization was assessed by means of (bio)leaching experiments (quantification by ICP-MS) as well as phytotoxicity (pot experiments). The alteration of pellets surface was identified by scanning electron microscopy (SEM-EDS).

We found that (bio)weathering by A. thiooxidans results in metal-enriched leachates (up to $1.75 \% \mathrm{Sb}, 0.8 \% \mathrm{As}$ and $0.1 \% \mathrm{~Pb}$ ). Pellet dissolution under biotic conditions was up to 6.5 times faster as compared to acidic abiotic conditions at which the release of elements did not exceed $0.3 \%$. We also observed that $\mathrm{Pb}$ release is mainly governed by $\mathrm{pH}$ variations rather than by bacterial activity itself. In contrast, the release of As and $\mathrm{Sb}$ is highly enhanced in the presence of bacteria. Furthermore, $\mathrm{Pb}$ pellets were found reactive when remaining in contact with organic solutions. The plant growth in soil impacted by pellets differs from that observed in control soil.

We conclude that as the result of (bio)weathering processes some adverse environmental impacts inherent to pellets dissolution can undoubtedly occur in wetland hunting areas.

Acknowledgements: This work was financially supported by the National Science Centre (NCN) in Poland in the frame of SONATA program under grant agreement UMO-2018/31/D/ST10/00738 to AP. 